\title{
Lattice Boltzmann method with restored Galilean invariance
}

\author{
N. I. Prasianakis, ${ }^{1, *}$ I. V. Karlin, ${ }^{2,3, \dagger}$ J. Mantzaras, ${ }^{1, \ddagger}$ and K. B. Boulouchos ${ }^{2, \S}$ \\ ${ }^{1}$ Combustion Research, Paul Scherrer Intsitute, 5232 Villigen PSI, Switzerland \\ ${ }^{2}$ Aerothermochemistry and Combustion Systems Lab, ETH Zurich, 8092 Zurich, Switzerland \\ ${ }^{3}$ School of Engineering Sciences, University of Southampton, SO17 1BJ Southampton, United Kingdom
}

(Received 4 December 2008; published 3 June 2009)

\begin{abstract}
An isothermal model on the standard two-dimension nine-velocity lattice (D2Q9) is proposed and analyzed. It originates from the thermal model with energy conservation introduced by N. I. Prasianakis and I. V. Karlin [Phys. Rev. E 76, 016702 (2007)]. The isothermal and the thermal equivalent models are tested through the simulation of the decay of a shear wave and of a temperature wave. Both are shown to be Galilean invariant, reference temperature independent, and rotational isotropic through the measurement of the transport coefficients on a rotated moving frame of reference.
\end{abstract}

DOI: 10.1103/PhysRevE.79.066702

PACS number(s): 47.11.-j, 05.20.Dd

\section{INTRODUCTION}

The lattice Boltzmann (LB) method has been used with a remarkable success as a powerful alternative tool for solving the hydrodynamic Navier-Stokes (NS) equations, with applications ranging from large Reynolds number flows to flows at the micron scale, porous media, and multiphase flows [1]. The LB method solves a fully discrete kinetic equation for populations $f_{i}(\boldsymbol{x}, t)$, designed in a way that it reproduces the Navier-Stokes equations in the hydrodynamic limit in $D$ dimensions. Populations correspond to discrete velocities $\boldsymbol{c}_{i}$ for $i=0,1, \ldots, Q-1$, which fit into a regular spatial lattice with the nodes $\boldsymbol{x}$. This enables a simple and highly efficient "stream-along-links-and-equilibrate-at-nodes" realization of the LB algorithm. The success of the LB method is mainly in the low Mach number isothermal NS limit. The most commonly used LB models are the ones that reside on the socalled standard lattices, which are characterized by a relatively small number of discrete velocities. Populations $f_{i}$ propagate only to their neighboring nodes during the streaming process. For $D=2$ the prevailing lattice is the twodimension nine-velocity lattice (D2Q9) and for $D=3$ the D3Q15, D3Q19, and D3Q27 lattices are usually implemented. The discrete velocity populations, at equilibrium state $f_{i}^{\text {eq }}$, are polynomial expressions of density $\rho$ and velocity $u$. At the hydrodynamic limit, the aforementioned models approximate within certain accuracy the target isothermal NS equations [1-4]. The absence of high order velocity terms, the geometric constraints of the regular lattice, and the small number of discrete velocities prohibit a fully correct description of hydrodynamics on standard lattices. Moreover, the method is restricted to a fixed working temperature $T_{0}=1 / 3$ in lattice units. Changing the working reference temperature or increasing the fluid velocity results in an increase in the deviation from the isothermal NS equations which indicates lack of Galilean invariance and strong reference temperature dependence.

\footnotetext{
*Corresponding author; nikolaos.prasianakis@psi.ch

${ }^{\dagger}$ karlin@lav.mavt.ethz.ch

¥ioannis.mantzaras@psi.ch

§boulouchos@lav.mavt.ethz.ch
}

In this paper, an isothermal model on the D2Q9 lattice is proposed. The new equilibrium populations are functions of density, working reference temperature, and velocity, and contain terms up to power four in velocity. This results in an enhanced description of the population higher-order moments, pertinent to the recovery of the isothermal NavierStokes equations. While the working reference temperature is allowed to change, deviation terms that are functions of the reference temperature $T_{0}$ and of the fluid velocity $u$ start to appear. These terms are identified and removed after introducing specifically designed counterterms. The Galilean invariance, reference temperature independence, and rotational isotropy of the proposed isothermal model are demonstrated through the simulation of the decay of a shear wave. In order to expose the deviation terms and demonstrate the efficiency of the compensation process, the wave vector of the flow is not aligned to the lattice grid, the lattice temperature is different from the usual value $T_{0}=1 / 3$ and the magnitude of the velocity of the frame of reference is altered accordingly. The measured quantity is the viscosity of the fluid, and comparison with the standard formulation for ideal gases, which is known to lack Galilean invariance and rotational isotropy, is conducted showing the advantages of the proposed approach.

The thermal model recently introduced in Refs. $[5,6]$ is also tested for the same qualities. This model is an energyconserving model that uses a single distribution function for simulation of flows on standard lattices. It has a broader range of applicability compared to the isothermal models since it allows the study of energy (heat) transfer related problems. The viscosity and thermal conductivity of the model are measured through the simulation of the decay of a shear wave and of a temperature wave on a moving frame of reference.

The outline of the paper is as follows: in Sec. II the standard isothermal LB model on the D2Q9 lattice is reviewed. In Sec. III, the guided equilibrium populations, which consist the basis of the proposed isothermal model, are presented. In Sec. IV, the deviation of the model presented in Sec. III, from the isothermal NS equations is identified and neutralized through a forcing procedure. In Sec. V, numerical simulations verify the theoretically predicted superiority of the current model over the standard LB method. In Sec. VI, the 
thermal model of Refs. [5,6] is studied in detail and simulation results are presented.

\section{STANDARD LATTICE BOLTZMANN MODELS ON THE D2Q9 LATTICE}

For the sake of presentation, we consider the popular nine-velocity model, the so-called D2Q9 lattice, for which the discrete velocities are: $\boldsymbol{c}_{0}=(0,0), \boldsymbol{c}_{i}=( \pm 1,0)$ and $(0, \pm 1)$ for $i=1-4$, and $c_{i}=( \pm 1, \pm 1)$, for $i=5-8$ [7]. Recall that the D2Q9 lattice derives from the three-point GaussHermite formula [8], with the following weights of the quadrature $w(-1)=1 / 6, w(0)=2 / 3$, and $w(+1)=1 / 6$. Let us arrange in the list $c_{x}$ all the components of the lattice velocities along the $x$ axis and similarly in the list $c_{y}$. Analogously let us arrange in the list $f$ all the populations $f_{i}$. Algebraic operations for the lists are always assumed component wise. The sum of all the elements of the list $p$ is denoted by $\langle p\rangle$ $=\sum_{i=0}^{Q-1} p_{i}$. In particular, the dimensionless density $\rho$, the flow velocity $\boldsymbol{u}$, and the second-order moment (pressure tensor) $\boldsymbol{P}$ are defined by $\rho=\langle f\rangle, \rho \boldsymbol{u}=\langle\boldsymbol{c} f\rangle$, and $P=\langle\boldsymbol{c} \boldsymbol{c} f\rangle$, respectively. The elements of the list $f^{\text {eq }}$ are

$$
\begin{aligned}
f_{i}^{\mathrm{eq}}= & \rho w_{i}\left[1+\frac{c_{i \alpha} u_{\alpha}}{c_{s}^{2}}+\frac{u_{\alpha} u_{\beta}}{2 c_{s}^{4}}\left(c_{i \alpha} c_{i \beta}-c_{s}^{2} \delta_{\alpha \beta}\right)\right. \\
& \left.+\theta \frac{u_{\alpha} u_{\beta} u_{\gamma} c_{i \gamma}}{6 c_{s}^{6}}\left(c_{i \alpha} c_{i \beta}-3 c_{s}^{2} \delta_{\alpha \beta}\right)\right]
\end{aligned}
$$

where $w_{i}=w_{i}\left(c_{x}\right) w_{i}\left(c_{y}\right)$, and $c_{s}=1 / \sqrt{3}$ is the speed of sound in lattice units. For $\theta=0$, Eq. (1) becomes the standard isothermal model [7]. This model lacks Galilean invariance, rotational isotropy, and the speed of sound is fixed to $c_{s}^{2}=1 / 3$. More specifically, there exist several cubic in velocity deviation terms that are insignificant as long as the flow velocity is relatively small [2]. When the flow velocity is increased these deviation terms are activated resulting in a wrong behavior, such as the viscosity dependency on the fluid velocity. For $\theta=1$, the equilibrium populations contain cubic terms in the velocity [4], intended to improve the lack of Galilean invariance of the standard D2Q9 LB model. However, improvement is achieved only in special cases, and a complete compensation of the deviation terms in the higher equilibrium moments is not possible $[3,4]$.

\section{GUIDED EQUILIBRIUM ON THE D2Q9 LATTICE}

In this paper, we introduce a different equilibrium for the LB models of isothermal flow. For that, we consider the entropy function of the D2Q9 lattice [9],

$$
H(f)=\langle f \ln (f / w)\rangle .
$$

In Refs. $[5,6]$, in order to derive the LB model for compressible flows, a guided equilibrium has been introduced as the minimizer of the entropy function (2) under the following set of constraints:

$$
\begin{gathered}
\left\langle f^{\mathrm{eq}}\right\rangle=\rho, \\
\left\langle\boldsymbol{c} f^{\mathrm{eq}}\right\rangle=\rho u,
\end{gathered}
$$

$$
\left\langle c^{2} f^{\mathrm{eq}}\right\rangle=2 \rho T+\rho u^{2},
$$

$$
\left\langle\boldsymbol{c c} f^{\mathrm{eq}}\right\rangle=\rho T 1+\rho u \boldsymbol{u},
$$

where $\rho=\langle f\rangle$ and $\rho \boldsymbol{u}=\langle\boldsymbol{c} f\rangle$ are the density and the momentum as above, and $T$ is the temperature defined through the twodimensional ideal-gas equation of state, $\left\langle(\boldsymbol{c}-\boldsymbol{u})^{2} f\right\rangle=2 \rho T$. Details of the derivation of the guided equilibrium $f^{\mathrm{eq}}(\rho, \mathbf{u}, T)$ as a minimizer of the entropy function (2) under constraints (3)-(6) can be found in Refs. $[5,6,10]$. The result is written in a compact form

$$
f^{\mathrm{eq}}=\rho \prod_{\alpha=x, y} \frac{\left(1-2 c_{\alpha}^{2}\right)}{2^{c_{\alpha}^{2}}}\left(c_{\alpha}^{2}-1+c_{\alpha} u_{\alpha}+u_{\alpha}^{2}+T\right) .
$$

A few remarks on the entropy minimization problem and its solution [Eq. (7)] are useful. When Eq. (2) is minimized under the density and momentum constraints alone, Eqs. (3) and (4), one obtains the equilibrium $[9,11]$. Once the equation of state constraint [Eq. (5)] is also taken into account, one arrives at the consistent LB equilibrium with energy conservation $f_{C}^{\mathrm{eq}}(\rho, \boldsymbol{u}, T)$ of Ref. [12]. Constraints (3)-(5) are the standard local equilibrium conditions which express local conservation of mass, momentum, and energy. The condition on the equilibrium pressure tensor $\boldsymbol{P}^{\mathrm{eq}}[\mathrm{Eq}$. (6)], in addition, stipulates the Maxwell-Boltzmann form of the latter. This is a necessary (but not sufficient) condition in order to recover the Navier-Stokes equations. Following the proposal of Refs. $[5,6,13,14]$, conditions concerning the kinetic moments (not local conservations) in the entropy minimization problem are termed guiding constraints. Note that not all the three constraints on the components of $P^{\mathrm{eq}}[\mathrm{Eq} .(6)]$ are independent: The constraint for the trace, $\operatorname{tr} \boldsymbol{P}^{\mathrm{eq}}=P_{x x}^{\mathrm{eq}}+P_{y y}^{\mathrm{eq}}$, is equivalent to Eq. (5). Moreover, the consistent LB equilibrium with energy conservation $f_{C}^{\mathrm{eq}}(\rho, \boldsymbol{u}, T)$ of Ref. [12] already implies the offdiagonal component of the pressure tensor in the form (6), that is, $\left\langle c_{x} c_{y} f_{C}^{\mathrm{eq}}\right\rangle=\rho u_{x} u_{y}$. Thus, viewed as a refinement of $f_{C}^{\mathrm{eq}}$, the guided equilibrium $f^{\mathrm{eq}}=f_{C}+\varphi$ can equivalently be formulated as a minimization of an "excess" entropy $H(\varphi)=\left\langle\left(f_{C}\right.\right.$ $\left.+\varphi) \ln \left[\left(f_{C}+\varphi\right) / w\right]\right\rangle$, with respect to $\varphi$ which satisfies homogeneous constraints,

$$
\left\langle\left\{1, c_{x}, c_{y}, c^{2}, c_{x} c_{y}\right\} \varphi\right\rangle=0,
$$

and a single nonhomogeneous condition on the normal stress difference,

$$
N^{\mathrm{eq}}=P_{x x}^{\mathrm{eq}}-P_{y y}^{\mathrm{eq}}=\left\langle\left(c_{x}^{2}-c_{y}^{2}\right)\left(f_{C}^{\mathrm{eq}}+\varphi\right)\right\rangle=\rho\left(u_{x}^{2}-u_{y}^{2}\right) .
$$

It is interesting to note that, while a perturbation method was used in Refs. [5,6], function (7) is in fact the exact minimizer of the entropy [Eq. (2)] under the specified constraints, as was recently pointed out in Ref. [15]. Function (7) is positive if the temperature $T$ is in the range $\delta T=\delta T_{x} \cap \delta T_{y}$, where $\delta T_{x, y}=\left\{T:\left|u_{x, y}\right|<T+u_{x, y}^{2}<1\right\}$, which is well satisfied at low Mach numbers, $|u| \ll 1$. In this case, $0 \leqq T \leqq 1$.

For the purpose of the present paper, the equilibrium for the isothermal flow simulation is read off the formula (7) upon fixing the temperature at a constant value $T=T_{0}=c_{s}^{2}$, 


$$
f^{\mathrm{eq}}=\rho \prod_{\alpha=x, y} \frac{\left(1-2 c_{\alpha}^{2}\right)}{2^{c_{\alpha}^{2}}}\left(c_{\alpha}^{2}-1+c_{\alpha} u_{\alpha}+u_{\alpha}^{2}+T_{0}\right) .
$$

where $T_{0} \in(0,1)$. Note that the reference temperature is not necessarily fixed at $T_{0}=1 / 3$. Equilibrium (8) is the starting point for our derivation of the LB model with restored Galilean invariance.

\section{RESTORING GALILEAN INVARIANCE}

We shall now analyze the higher-order moments of the equilibrium (8) pertinent to recovering the Navier-Stokes equations at a fixed temperature. After that, the remaining deviations in the higher-order moments will be cured upon introducing counterterms into the kinetic equation. The procedure closely follows Refs. [5,6] where the compressible flow was considered.

Besides the equilibrium pressure tensor $\boldsymbol{P}^{\mathrm{eq}}$ already discussed, also the equilibrium third-order moments,

$$
Q^{\mathrm{eq}}=\left\langle\boldsymbol{c c c} f^{\mathrm{eq}}\right\rangle,
$$

need to be in the Maxwell-Boltzmann (MB) form in order to recover the Navier-Stokes equations at a fixed temperature. The Maxwell-Boltzmann formula $Q^{\mathrm{MB}}$ is known, and is presented here in a component form

$$
Q_{\alpha \beta \gamma}^{\mathrm{MB}}=\rho T_{0}\left(u_{\alpha} \delta_{\beta \gamma}+u_{\beta} \delta_{\alpha \gamma}+u_{\gamma} \delta_{\alpha \beta}\right)+\rho u_{\alpha} u_{\beta} u_{\gamma} .
$$

Note that, by construction of the guided equilibrium, the equilibrium pressure tensor $\boldsymbol{P}^{\text {eq }}$ is already consistent with the Maxwell-Boltzmann form [see Eq. (6)]. Moreover, the offdiagonal elements of the third-order moment, $Q_{x x y}^{\mathrm{eq}}$ and $Q_{y y x}^{\mathrm{eq}}$, also satisfy the Maxwell-Boltzmann relations [Eq. (10)] exactly. This is a property missing from the standard formulation. The remaining deviation is contained in the diagonal components of the third-order moment [Eq. (9)]. Denoting $\Delta Q_{\alpha \alpha \alpha}=Q_{\alpha \alpha \alpha}^{\mathrm{eq}}-Q_{\alpha \alpha \alpha}^{\mathrm{MB}}$, we have

$$
\Delta Q_{\alpha \alpha \alpha}=\rho u_{\alpha}\left(1-3 T_{0}\right)-\rho u_{\alpha}^{3} .
$$

Note that, for the reference temperature $T_{0}=1 / 3$, the first term annihilates in Eq. (11), and the deviation is given by the remaining $O\left(u^{3}\right)$ terms.

When the guided equilibrium (8) is used in the Boltzmann Bhatnagar-Gross-Krook (BGK) equation [16],

$$
\partial_{t} f+c \cdot \nabla f=-\frac{1}{\tau}\left(f-f^{\mathrm{eq}}\right),
$$

where $\nabla=\partial / \partial \boldsymbol{x}$, and $\tau>0$ is the relaxation time, the deviation [Eq. (11)] results in spurious terms in the hydrodynamic limit at the level of the Navier-Stokes equation. More specifically, using the standard Chapman-Enskog method in order to derive the Navier-Stokes equation from Eq. (12), the terms appearing in the momentum equation have the form of a force $\boldsymbol{F}$,

$$
\begin{aligned}
& F_{x}=\tau \partial_{x}^{2}\left(\rho u_{x}\left(1-3 T_{0}\right)-\rho u_{x}^{3}\right), \\
& F_{y}=\tau \partial_{y}^{2}\left(\rho u_{y}\left(1-3 T_{0}\right)-\rho u_{y}^{3}\right) .
\end{aligned}
$$

This has a negative effect on the accuracy of the simulations, especially whenever the temperature deviates from the value
$T_{0}=1 / 3$, and/or when the magnitude of the fluid velocity is increased (see Sec. V). Note that this deviation term is different from the deviation term that appears in the momentum equation of the thermal model of Ref. [5].

In order to alleviate this limitation, the implementation of the forcing as presented in [5] is used. The counterterm $\Psi$ is introduced into the Boltzmann BGK kinetic equation [Eq. (12)],

$$
\partial_{t} f+c \cdot \nabla f=-\frac{1}{\tau}\left(f-f^{\mathrm{eq}}\right)+\Psi
$$

where the term $\Psi$ is responsible for the correction in the macroscopic momentum equation. Function $\Psi$ is defined using vectors $\boldsymbol{\Psi}$,

$$
\begin{aligned}
& \Psi_{x}=(1 / 4)[0,4,0,-4,0,-1,1,1,-1], \\
& \Psi_{y}=(1 / 4)[0,0,4,0,-4,-1,-1,1,1],
\end{aligned}
$$

so that the contribution of the correction term occurs only at the momentum equation,

$$
\Psi=-\boldsymbol{\Psi} \cdot \boldsymbol{F} .
$$

The introduction of counterterms results in a Galilean invariant, reference temperature independent and rotational isotropic isothermal model, on the D2Q9 lattice. The macroscopic limit of this model is the isothermal Navier-Stokes equations,

$$
\begin{gathered}
\partial_{t} \rho=-\nabla \cdot(\rho \boldsymbol{u}), \\
\partial_{t} \boldsymbol{u}=-\boldsymbol{u} \cdot \nabla \boldsymbol{u}-\frac{1}{\rho} \nabla\left(\rho T_{0}\right)+\frac{1}{\rho} \nabla \cdot\left[\mu\left(\nabla \boldsymbol{u}+\nabla \boldsymbol{u}^{T}\right)\right],
\end{gathered}
$$

where $\mu=\tau \rho T_{0}$ is the dynamic viscosity. Note that a change in the reference temperature allows to adjust consistently the speed of sound $\left(c_{s}=\sqrt{T_{0}}\right)$. Turning the kinetic Eq. (15) into a numerical LB scheme follows Refs. [5,6]. For convenience, all necessary formulas are collected in the Appendix.

At this point, it should be noted that the alteration of the lattice BGK equation in order to obtain specific macroscopic dynamics, has been used in the field of multiphase LB modeling [17-20]. In Ref. [20], a one-dimension multiphase model is presented and the non-ideal-gas behavior is enforced through the addition of forcing terms. Among other, the terms $\partial_{\beta} \partial_{\gamma}\left(\rho u_{a} u_{\beta} u_{\gamma}\right)$ are removed from the momentum equation. The isothermal model presented in this paper and the multiphase model presented in Ref. [20] have different origin, different purpose and hence the correction terms as well as their implementation is different. More specific, the model of Ref. [20] is based on the properties of the standard isothermal model with fixed temperature $T_{0}=1 / 3$ in lattice units. Consequently, if this model is used for single phase ideal-gas flow simulations, the stress tensor $P_{\alpha \beta}^{\mathrm{eq}}$ and the ideal-gas law will be accurate only at $T_{0}=1 / 3$. The thirdorder moments $Q_{\alpha \beta \gamma}^{\mathrm{eq}}$ will contain only linear in velocity correct terms at this specific reference temperature. A change in the working temperature is not predicted, therefore at a working temperature different than the usual $T_{0}=1 / 3$, the equilibrium stress tensor $P_{\alpha \beta}^{\mathrm{eq}}$ will include wrong values of 
the static pressure and the ideal-gas law must be enforced. In addition, all the terms in all the third-order moments $Q_{\alpha \beta \gamma}^{\mathrm{eq}}$ will be inaccurate and the viscosity will always be equal to $\mu=\tau \rho / 3$ rather than $\mu=\tau \rho T_{0}$ as predicted by the kinetic theory. Contrary, for the isothermal model presented in this paper a change in the working temperature is allowed. The stress tensor $P_{\alpha \beta}^{\mathrm{eq}}$ is exact and the ideal-gas law is built-in by construction and thus always verified. Moreover, only the diagonal elements $Q_{x x x}^{\mathrm{eq}}$ and $Q_{y y y}^{\mathrm{eq}}$ are not exact as shown in Eq. (11), giving rise to the deviation terms of Eq. (13), and the viscosity value is in agreement with the kinetic theory.

We shall now proceed with a numerical validation of the proposed flow solver. In Sec. V, the isothermal model proposed in this section is validated. In Sec. VI, the thermal model of Refs. [5,6] is checked for Galilean invariance, reference temperature independence and rotational isotropy.

\section{MEASURING GALILEAN INVARIANCE, REFERENCE TEMPERATURE INDEPENDENCE, AND ISOTROPY OF ISOTHERMAL MODELS}

In this section, we shall first test the Galilean invariance, isotropy, and reference temperature independence of the isothermal LB model of Sec. IV. For that, we consider the simulation of the decay of a plain shear wave $[3,4,21]$. Galilean invariance can be verified by altering the reference frame velocity, while isotropy can be verified through the simulation of the flow in an inclined domain. The reference temperature independence can be verified by changing the reference temperature of the simulations.

For the lattice-aligned case, the wave vector of the shear wave is aligned along the $x$ direction of the computational grid. The initial conditions that characterize the flow are

$$
\rho(0)=\rho_{0}, \quad u_{x}(0)=U_{0 x}+a_{0} \sin (2 \pi y / L), \quad u_{y}(0)=U_{0 y},
$$

where $U_{0 x}$ and $U_{0 y}$ represent the frame velocity components, and $a_{0}$ is the initial amplitude of the shear wave. Periodic boundary conditions are applied along the $x$ and $y$ axes as shown in the left picture of Fig. 1. Viscosity of the simulated fluid can be measured numerically through the time decay of the flow. Then, the measured viscosity can be compared with the originally imposed viscosity. Simulation results for this flow setup are illustrated in Fig. 2. For the simulations, $U_{0 x}$ $=0, L=100$ nodes on the $y$ axis, the amplitude is set to $a_{0}$ $=0.001$, the speed of sound squared is $c_{s}^{2}=T_{0}$ with $T_{0}=1 / 3$, and the Mach number is defined as $\mathrm{Ma}=U_{0 y} / c_{s}$. For the standard model of Eq. (1) with $\theta=0$, an increase in the frame velocity results in a decrease in the measured viscosity. Contrary, the model of Eq. (1) with $\theta=1$, for this flow orientation, does not show unphysical behavior as has been also noticed in Ref. [3]. The same result is obtained for the isothermal model of Eq. (8) and the thermal model of Eq. (7) without correction terms implementation. The measured viscosity is independent of the frame velocity. This is theoretically expected since by the definition of the flow, the deviation terms $\partial_{x} \rho u_{x}^{3}$ and $\partial_{y} \rho u_{y}^{3}$ vanish.

In order to activate the deviation terms of Eq. (13), the wave vector of the flow is rotated by $\pi / 4$ as shown in the

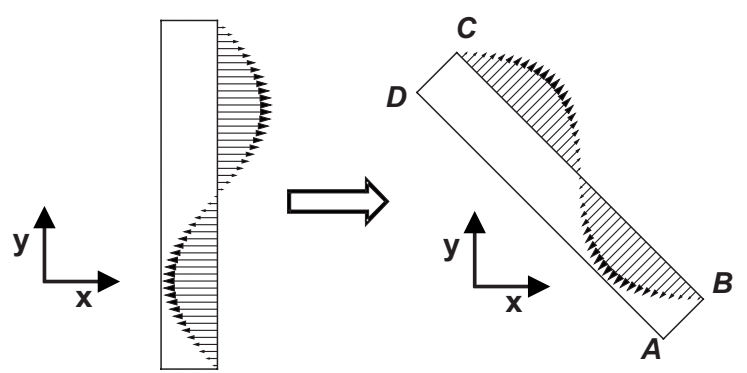

FIG. 1. Left: aligned setup. The wave vector of the flow is parallel to the $x$ axis of the lattice. Incomplete Galilean invariance of the standard isothermal model of Eq. (1) $(\theta=0)$ manifests itself as shown in Fig. 2, and in Ref. [3]. For the model of Eq. (1) $(\theta=1)$, for this specific flow setup and orientation, the deviation terms in the momentum equation are not active and unphysical behavior cannot be observed. Right: tilted setup. In order to activate the deviation terms in the momentum equation the wave vector of the flow is rotated by $\pi / 4$. Incomplete Galilean invariance of Eq. (1) with $(\theta=1)$, Eqs. (7) and (8) are now revealed.

right picture of Fig. 1. Periodic boundary conditions are applied between the interface $A D$ and $B C$, as well as between the interface $A B$ and $C D$, while in the lattice-aligned setup periodicity was applied along the $x$ and $y$ axes, respectively. From the theoretical point of view, the deviation terms $\partial_{x} \rho u_{x}^{3}$ and $\partial_{y} \rho u_{y}^{3}$ are now triggered. As expected, the models of Eqs. (1), (7), and (8) reveal their lack of Galilean and rotational invariance as the frame velocity increases. The initial conditions of the flow are

$$
\rho(0)=\rho_{0},
$$

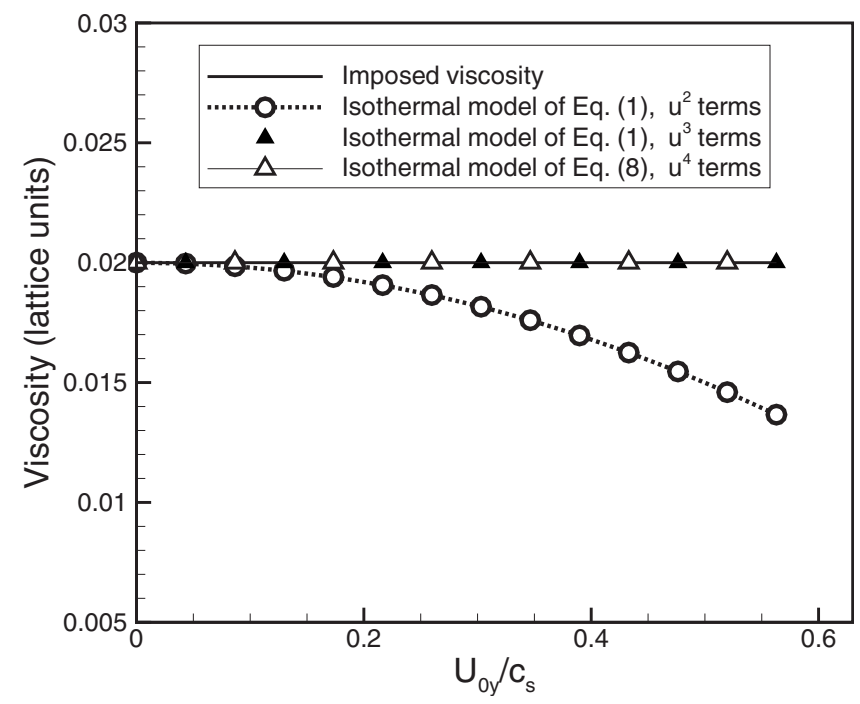

FIG. 2. Lattice-aligned setup. Numerical measurement of the fluid viscosity (lattice units) through the exponential decay of a shear wave. Solid line represents the imposed viscosity; circles and the dashed line represent the isothermal D2Q9 model of Eq. (1) with $\theta=0$ ( $u^{2}$ polynomials); Lack of Galilean invariance is evident; filled triangles represent the isothermal D2Q9 model of Eq. (1) with $\theta=1$ ( $u^{3}$ polynomials); empty triangles represent the isothermal D2Q9 model of Eq. (8) ( $u^{4}$ polynomials). 
TABLE I. Difference between the values of the imposed viscosity and the values of the viscosity measured from the simulations. For the model of Eq. (8) with counterterms implementation the difference remains at low levels as illustrated in Fig. 3

\begin{tabular}{ccc}
\hline \hline $\begin{array}{c}\text { Mach number } \\
\text { of the flow }\end{array}$ & $\begin{array}{c}\text { Eq. (8) with counter terms } \\
\frac{\left(\mu-\mu_{\text {sim }}\right)}{\mu} \times 100\end{array}$ & $\begin{array}{c}\text { Eq. (1) with } \theta=1 \\
\frac{\left(\mu-\mu_{\text {sim }}\right)}{\mu} \times 100\end{array}$ \\
\hline 0.0 & $0.010 \%$ & $0.010 \%$ \\
0.2 & $0.011 \%$ & $3.0 \%$ \\
0.4 & $0.012 \%$ & $12 \%$ \\
0.6 & $0.015 \%$ & $27 \%$ \\
0.8 & $0.022 \%$ & $48 \%$ \\
\hline \hline
\end{tabular}

$$
\begin{aligned}
& u_{x}(0)=U_{0 x}+a_{0} \sin \left[\frac{2 \pi}{L \sqrt{2}}(-x+y)\right], \\
& u_{y}(0)=U_{0 y}+a_{0} \sin \left[\frac{2 \pi}{L \sqrt{2}}(-x+y)\right] .
\end{aligned}
$$

Similar to the lattice-aligned flow, the viscosity of the fluid is measured numerically. Simulation results for velocity $U_{0 x}$ equal to $U_{0 y}$, and $U_{0}=\left(U_{0 x}^{2}+U_{0 y}^{2}\right)^{1 / 2}$, are presented in Table I and Fig. 3. The model of Eq. (1) with $\theta=1$, exhibits large deviation as soon as the Mach number of the flow increases above zero. The guided equilibrium models of Eqs. (7) and (8) without the forcing term implementation show the same behavior. In the same plot, the results using the compensation procedure, as presented in Sec. IV for the model of Eq. (8), are shown. As expected, the simulation results of the

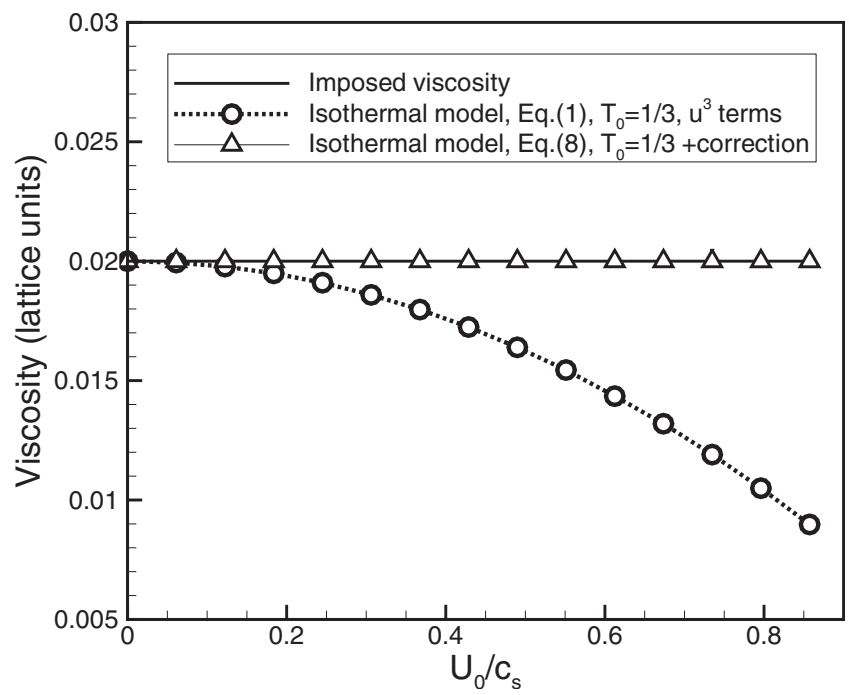

FIG. 3. Rotated flow field. Numerical measurement of the fluid viscosity (lattice units) through the exponential decay of a shear wave. Solid line represents the imposed viscosity. Circles and the dashed line represent the isothermal D2Q9 model of Eq. (1) ( $u^{3}$ polynomials), lack of Galilean invariance is revealed; triangles represent the simulation results of the forced isothermal model as presented in Sec. IV, Galilean invariance and rotational isotropy are restored.

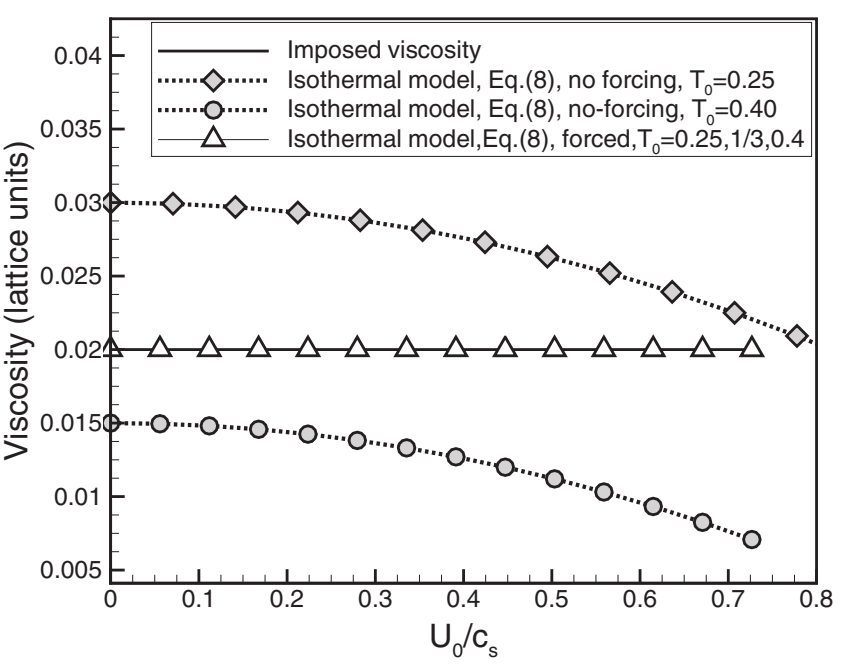

FIG. 4. Rotated flow field. Numerical measurement of the fluid viscosity (lattice units) through the exponential decay of a shear wave. Solid line represents the imposed viscosity; circles and the dashed line represent the bare isothermal model of Eq. (8), operated at a reference temperature $T_{0}=0.4$ in lattice units; diamonds and the dashed line represent the bare isothermal model of Eq. (8), operated at a reference temperature $T_{0}=0.25$ in lattice units; triangles represent the simulation results of the forced isothermal model presented in Sec. IV for reference temperatures $T_{0}=0.25, T_{0}=1 / 3$, and $T_{0}$ $=0.4$.

forced isothermal model are always correct, even when the Mach number of the flow is relatively high. Galilean invariance and rotational isotropy have been restored. The same results can be obtained using the thermal model of Ref. [5]. Concerning the computational time, the isothermal guided equilibrium model with the application of the compensation procedure leads to a small computational overhead $(15 \%)$ compared to the standard isothermal D2Q9 formulation.

Since the deviation of the guided isothermal equilibrium model has been fully identified, it is now possible to change the working reference temperature $T_{0}$, which was always equal to $T_{0}=1 / 3$, for all the isothermal D2Q9 models. Changing the working reference temperature gives rise to the deviation terms which are multiplied by $\left(1-3 T_{0}\right)$, as can be deduced from Eq. (13). Consequently, the measured viscosity is not equal to the imposed one, even for the zero frame velocity case, revealing reference temperature dependence. The model should not be operated, at reference temperature other than $T_{0}=1 / 3$, without the implementation of the correction terms. The results for reference temperature values $T_{0}=0.25$ and $T_{0}=0.4$, whereas $U_{0 x}$ is equal to $U_{0 y}$ are demonstrated in Fig. 4. For all the simulations, the imposed viscosity is equal to $\mu=0.02$ in lattice units, while the reference temperature is varied. To avoid confusion, it should be noted that in these simulations a change in the reference temperature $T_{0}$ results in a change in the relaxation time through the formula $\tau=\mu /\left(\rho T_{0}\right)$ since the viscosity has a fixed value. The same results are obtained when the guided thermal equilibrium model without forcing terms Eq. (7) is implemented. After applying the forcing procedure, the measured viscosity is equal to the imposed one for different values of reference temperature and frame velocity. 


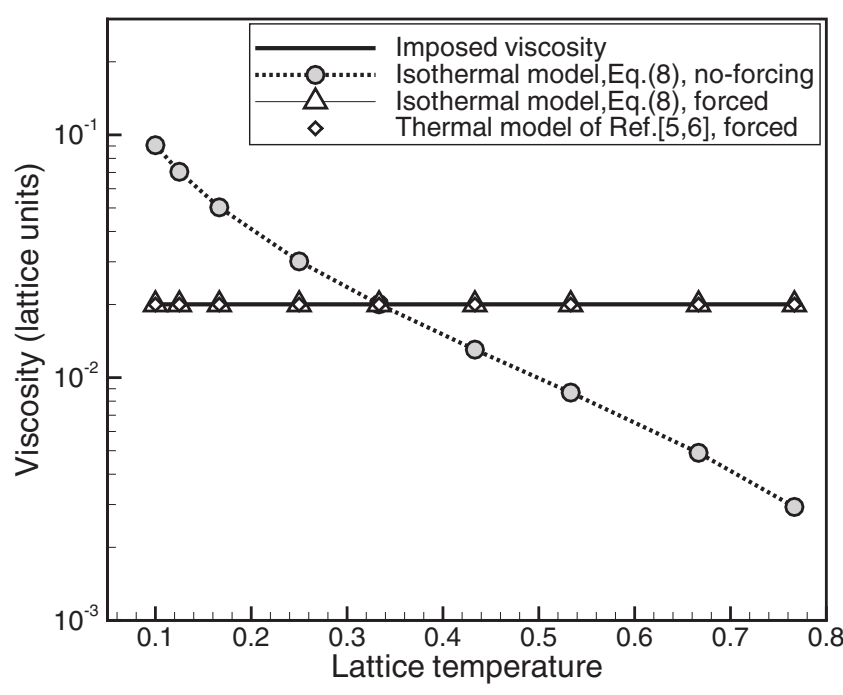

FIG. 5. Effect of change in the reference temperature. Numerical measurement of the fluid viscosity (lattice units) through the exponential decay of a shear wave. Solid line represents the imposed viscosity; circles and the dashed line represent the bare isothermal model presented in this paper while operated at different reference temperatures. Triangles represent the simulation results of the forced isothermal model presented in Sec. IV, and diamonds are the results of the forced thermal model of Refs. [5,6].

The effect of reference temperature is plotted for a wide range of temperature values in Fig. 5. Large deviations in the measured viscosity appear as soon as the reference temperature deviates from the value $T_{0}=1 / 3$. The bare thermal and isothermal models of Eqs. (7) and (8), respectively, produce identical results. Only at $T_{0}=1 / 3$ and for zero frame velocity, the models are able to reproduce the correct value of the viscosity. After applying the compensation procedure of Sec. IV, with appropriate correction terms, the reference temperature independence can be obtained for both cases as depicted in Fig. 5.

\section{MEASURING GALILEAN INVARIANCE, REFERENCE TEMPERATURE INDEPENDENCE, AND ISOTROPY OF THE THERMAL MODEL}

Finally, the decay of a temperature wave on a moving frame of reference is simulated. In combination with the simulation of the shear wave decay, it allows testing the thermal D2Q9 model of Ref. [5] for Galilean invariance, rotation isotropy, and reference temperature independence. The temperature wave decays exponentially at a rate defined by the thermal conductivity of the model $[21,22]$. The initial conditions for the lattice-aligned setup are

$$
\begin{gathered}
\rho(0)=\rho_{0}+a_{0} \sin \left(\frac{2 \pi}{L} y\right), \\
u_{x}(0)=U_{0 x}, \quad u_{y}(0)=0, \quad T(0)=\frac{\rho_{0} T_{0}}{\rho(0)},
\end{gathered}
$$

where $U_{0 x}$ stands for the frame velocity, $\alpha_{0}$ is the initial amplitude of the density field set as $a_{0}=0.001, T_{0}$ is a refer-

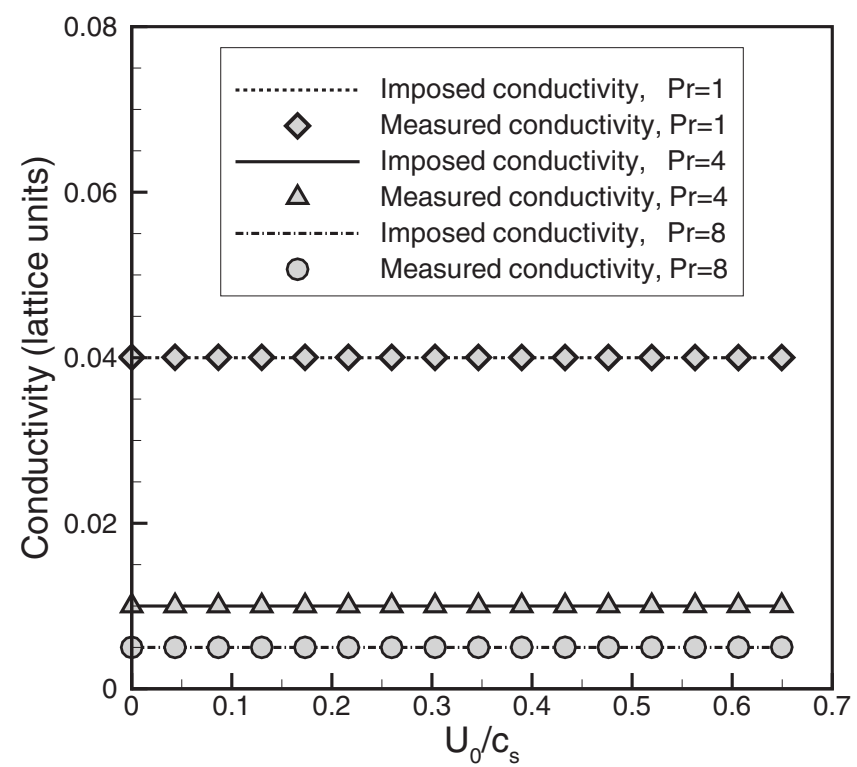

FIG. 6. Simulation results for the thermal conductivity (lattice units) for different values of Prandtl number. Lines represent the imposed conductivity while symbols represent the simulation results. The thermal D2Q9 model of Ref. [23] is implemented.

ence temperature, and $\rho_{0}$ is the characteristic density which is equal to unity for the current simulation. The static pressure is constant throughout the domain. For the $\pi / 4$ orientation of Fig. 1 the initial conditions are

$$
\begin{gathered}
\rho(0)=\rho_{0}+\alpha_{0} \sin \left(\frac{2 \pi}{L \sqrt{2}}(-x+y)\right), \\
u_{x}(0)=U_{0 x}, \quad u_{y}(0)=U_{0 y}, \quad T(0)=\frac{\rho_{0} T_{0}}{\rho(0)},
\end{gathered}
$$

where $U_{0 x}=U_{0 y}$ in order to maintain zero velocity of the fluid perpendicular to the wave vector of the temperature wave. This is required in order to have a simple exponential decay of the temperature profile [21]. The thermal conductivity of the simulated fluid can be measured numerically through the exponential decay of the temperature profile. In Fig. 6, the measured conductivity and the imposed conductivity are plotted for three different values of the Prandtl number ( $\mathrm{Pr}$ $=[1,4,8])$. It is reminded that the bare guided equilibrium thermal model of Eq. (7) comes with a fixed Prandtl value $\operatorname{Pr}=4$ [23], and that the forcing procedure allows to change the Prandtl number of the simulated fluid [5]. The imposed viscosity is $\mu=0.02$ in lattice units, resulting in a thermal conductivity equal to $\kappa=c_{p} \mu / \operatorname{Pr}$. The numerically measured conductivity is in excellent agreement with the imposed one.

\section{CONCLUSION}

In this paper, a isothermal D2Q9 model that offers the flexibility to alter the working reference temperature has been presented. Deviation terms that are found to be functions of the working reference temperature and of the fluid velocity have been identified and neutralized. Comparison 
tests with the most commonly used formulations suggest that the proposed model removes the low Mach number limitation as well as the frozen reference temperature condition. This drastically enhances the accuracy of the lattice Boltzmann method. It is demonstrated that unlike previous models on the standard D2Q9 lattice, this model after applying a forcing procedure becomes Galilean invariant, reference temperature independent, and rotational isotropic. The computational overhead of the forced model presented in this paper is small compared to the standard isothermal formulation. This model can be considered as a better basis model for the development of more complex lattice Boltzmann models.

The forced thermal model, introduced in Ref. [5], is a single distribution function model on the minimal D2Q9 lattice. In this paper, it is shown that this model is also Galilean invariant, reference temperature independent, and rotational isotropic after measuring the transport coefficients (viscosity and conductivity) in specific benchmark flows. The extension of this class of models in three dimensions is straightforward.

\section{ACKNOWLEDGMENTS}

We acknowledge the support of CCEM-CH. I.V.K. thanks A. Wagner for useful discussion of Ref. [20].

\section{APPENDIX: THE LATTICE BOLTZMANN SCHEME}

Following Refs. [5,6], the lattice Bhatnagar-Gross-Krook (BGK) equation with a transformation similar to the one introduced in [24] reads

$$
g=f+\frac{\delta t}{2 \tau}\left(f-f^{\mathrm{eq}}\right)-\frac{\delta t}{2} \Psi,
$$

$$
g_{t+\delta t}=g_{t}+\frac{2 \delta t}{\delta t+2 \tau}\left[f_{t}^{\mathrm{eq}}-g_{t}\right]+\frac{2 \tau \delta t}{\delta t+2 \tau} \Psi_{t},
$$

where the time step $\delta t$ is set $\delta t=1$ below. Computation is carried out on the level of the transformed populations $g$. The equilibrium populations $f^{\text {eq }}$, needed in Eq. (A2), are computed with the help of the moments of Eq. (A1), which relate the locally conserved moments of the $f$ populations $f$ with the moments of the $g$ populations. It is straightforward to show that the density $\rho(f)$ and the velocity $\boldsymbol{u}$ are evaluated as follows:

$$
\rho(f)=\rho(g), \quad \boldsymbol{u}(f)=\boldsymbol{u}(g)-\frac{\delta t}{2 \rho} \boldsymbol{F} .
$$

The lattice Boltzmann scheme of Eq. (A2) is semiexplicit due to the presence of the correction terms $\Psi_{i}$ (and not fully explicit, as in the standard case without any corrections). The following algorithm is implemented for the collision step,

Step 1. Calculate $\rho, \boldsymbol{u}$ using Eq. (A3), $\boldsymbol{F}^{t-1}$ and $g_{t}$ values.

Step 2. Calculate $\boldsymbol{F}^{t}$ using values of $\rho$ and $\boldsymbol{u}$ from step 1.

Step 3. Calculate $\rho$ and $\boldsymbol{u}$ using Eq. (A3) and $g_{t}$ and the values calculated in step 2 .

Step 4. Use $\rho$ and $\boldsymbol{u}$ from step 3 for the calculation of the equilibrium values [Eq. (1)].

Step 5. Use $\boldsymbol{F}^{t}$ from step 2 in the discrete Eq. (A2) along with the equilibrium values calculated in step 4 .

Spatial derivatives in the correction term $\boldsymbol{F}$ can be evaluated using a second-order central difference scheme: At a node $(i, j)$, where $i$ and $j$ correspond to the $x$ and $y$ directions, respectively, we have

$$
\begin{aligned}
& F_{x}^{(i, j)}=\tau\left[\Delta Q_{x x x}^{(i-1, j)}-2 \Delta Q_{x x x}^{(i, j)}+\Delta Q_{x x x}^{(i+1, j)}\right], \\
& F_{y}^{(i, j)}=\tau\left[\Delta Q_{x x x}^{(i, j-1)}-2 \Delta Q_{x x x}^{(i, j)}+\Delta Q_{x x x}^{(i, j+1)}\right],
\end{aligned}
$$

where $\Delta Q_{\alpha \alpha \alpha}$ are given by Eq. (11).
[1] S. Succi, The Lattice Boltzmann Equation for Fluid Dynamics and Beyond (Oxford University Press, New York, 2001).

[2] Y. H. Qian and S. Orszag, Europhys. Lett. 21, 255 (1993).

[3] G. Házi and P. Kávrán, J. Phys. A 39, 3127 (2006).

[4] Y. H. Qian and Y. Zhou, Europhys. Lett. 42, 359 (1998).

[5] N. I. Prasianakis and I. V. Karlin, Phys. Rev. E 76, 016702 (2007).

[6] N. I. Prasianakis and I. V. Karlin, Phys. Rev. E 78, 016704 (2008).

[7] Y. H. Qian, D. d'Humieres, and P. Lallemand, Europhys. Lett. 17, 479 (1992).

[8] X. Shan and X. He, Phys. Rev. Lett. 80, 65 (1998).

[9] I. V. Karlin, A. Ferrante, and H. C. Öttinger, Europhys. Lett. 47, 182 (1999).

[10] N. I. Prasianakis, Ph.D. thesis No. 17739, ETH Zurich, 2008.

[11] S. Ansumali, I. V. Karlin, and H. C. Öttinger, Europhys. Lett. 63, 798 (2003).

[12] S. Ansumali and I. V. Karlin, Phys. Rev. Lett. 95, 260605 (2005).

[13] I. V. Karlin and S. Succi, Phys. Rev. E 58, R4053 (1998).
[14] S. Succi, I. V. Karlin, and H. Chen, Rev. Mod. Phys. 74, 1203 (2002).

[15] P. Asinari and I. V. Karlin, Phys. Rev. E 79, 036703 (2009).

[16] P. L. Bhatnagar, E. P. Gross, and M. Krook, Phys. Rev. 94, 511 (1954).

[17] M. R. Swift, W. R. Osborn, and J. M. Yeomans, Phys. Rev. Lett. 75, 830 (1995).

[18] D. Holdych, D. Rovas, J. Georgiadis, and R. Buckius, Int. J. Mod. Phys. C 9, 1393 (1998).

[19] T. Inamuro, N. Konishi, and F. Ogino, Comput. Phys. Commun. 129, 32 (2000).

[20] A. Wagner and Q. Li, Physica A 362, 105 (2006).

[21] X. B. Nie, X. Shan, and H. Chen, Europhys. Lett. 81, 34005 (2008).

[22] X. Shan and H. Chen, Int. J. Mod. Phys. C 18, 635 (2007).

[23] N. I. Prasianakis and K. B. Boulouchos, Int. J. Mod. Phys. C 18, 602 (2007).

[24] X. He, S. Chen, and G. D. Doolen, J. Comput. Phys. 146, 282 (1998). 